\title{
Literatur
}

\section{Gesellschaftsanalyse in Zeiten der Pandemie}

Volkmer, Michael/Werner, Karin (Hrsg.) 2020: Die Corona-Gesellschaft. Analysen zur Lage und Perspektiven für die Zukunft. Transcript.

Besprochen von Hans Jonas Gunzelmann, Scuola Normale Superiore, Palazzo Strozzi, 50123 Florenz, Italien, E-Mail: hans.gunzelmann@sns.it

https://doi.org/10.1515/fjsb-2021-0030

$\mathrm{Zu}$ Beginn der Corona-Pandemie wurde die öffentliche Debatte von virologischen und epidemiologischen Beiträgen dominiert, während sozial- und kulturwissenschaftliche Arbeiten zunächst eine randständige Rolle einnahmen. Der Sammelband „Die Corona-Gesellschaft. Analysen zur Lage und Perspektiven für die Zukunft“, der von Michael Volkmer und Karin Werner im Juli 2020 im TranscriptVerlag herausgegeben wurde, hat sich zum Ziel gesetzt, eben solche geistes- und gesellschaftswissenschaftlichen Beiträge in die Debatte einzubringen. Der Band bietet Bewegungsforscher*innen und anderen Interessierten einen guten Überblick, wenngleich er im Lichte der dynamischen Pandemielage und des frühen Veröffentlichungsdatums im Sommer 2020 gelesen werden sollten.

\section{Perspektiven auf die Pandemie}

Mit zunehmender Ausbreitung des Coronavirus im Frühjahr 2020 wurde schnell deutlich, dass die Pandemie umfassende Konsequenzen für nahezu alle Lebensbereiche haben würde. Die Struktur des Sammelbandes spiegelt diese Breite an Auswirkungen wider: Die Herausgeberinnen haben 39 jeweils etwa zehnseitige Beiträge in zwölf Themenfeldern gebündelt, die sich vor allem den verschiedenen disziplinären Perspektiven auf den Gegenstand widmen: so finden sich neben raum- und körpersoziologischen Texten historische Vergleiche, sowie politikwissenschaftliche und ökonomische Einordnungen. Diese werden abgerundet durch eher prospektive Beiträge zur Krisenbewältigung und zu „konkreten Utopien“.

Wer nun erste empirische Forschungsergebnisse oder theoretische Einlassungen zur Pandemie erwartet, wird allerdings enttäuscht werden. Aufgrund des frühen Veröffentlichungstermins im Juli 2020, also gut ein halbes Jahr nach dem ersten Nachweis eines Coronafalls in Deutschland, sind die Texte als essayistische Annäherungen an das bis dato neuartige Phänomen „Corona“ zu verstehen. Auch wenn es der erklärte Anspruch der Herausgeber*innen ist, „das Feuilleton-Niveau 
deutlich $\mathrm{zu}$ überschreiten und die aktuelle Lage mit wissenschaftlicher Elle zu messen“ (13), so sind tiefergehende Erkenntnisse durch die Kürze der einzelnen Beiträge freilich nicht möglich. Gleichzeitig besteht in dem Format, kurze, aber viele verschiedene Perspektiven zu bündeln, der entscheidende Mehrwert des Sammelbandes. Schließlich erfordert es die Neuartigkeit der Pandemie und damit das Nicht-Wissen darüber, Analyseversuche aus möglichst unterschiedlichen Richtungen vorzunehmen, anstatt eine singuläre Herangehensweise zu privilegieren. Die daraus resultierenden einzelnen Essays werden der eingangs erwähnten Spannbreite der Corona-Auswirkungen durchaus gerecht.

\section{Krise oder Kontinuität?}

Auch wenn sich die einzelnen Beiträge sehr unterschiedlichen Aspekten der Pandemie widmen, so ist ihnen doch gemein, dass sie die Pandemie nicht als isolierten Untersuchungsgegenstand betrachten, sondern im engen Zusammenhang mit bereits bestehenden sozialen Strukturen, Praktiken und Prozessen. Diese zunächst recht allgemeine Aussage zieht sich in zwei roten Fäden durch den Band. Zum einen wird wiederholt betont, dass das Virus selbst zwar nicht diskriminiere, aber dennoch nicht der Gleichmacher sei, zu dem es zu Beginn in den Medien verklärt wurde. Im Gegenteil spüle die Pandemie „drastisch auch die

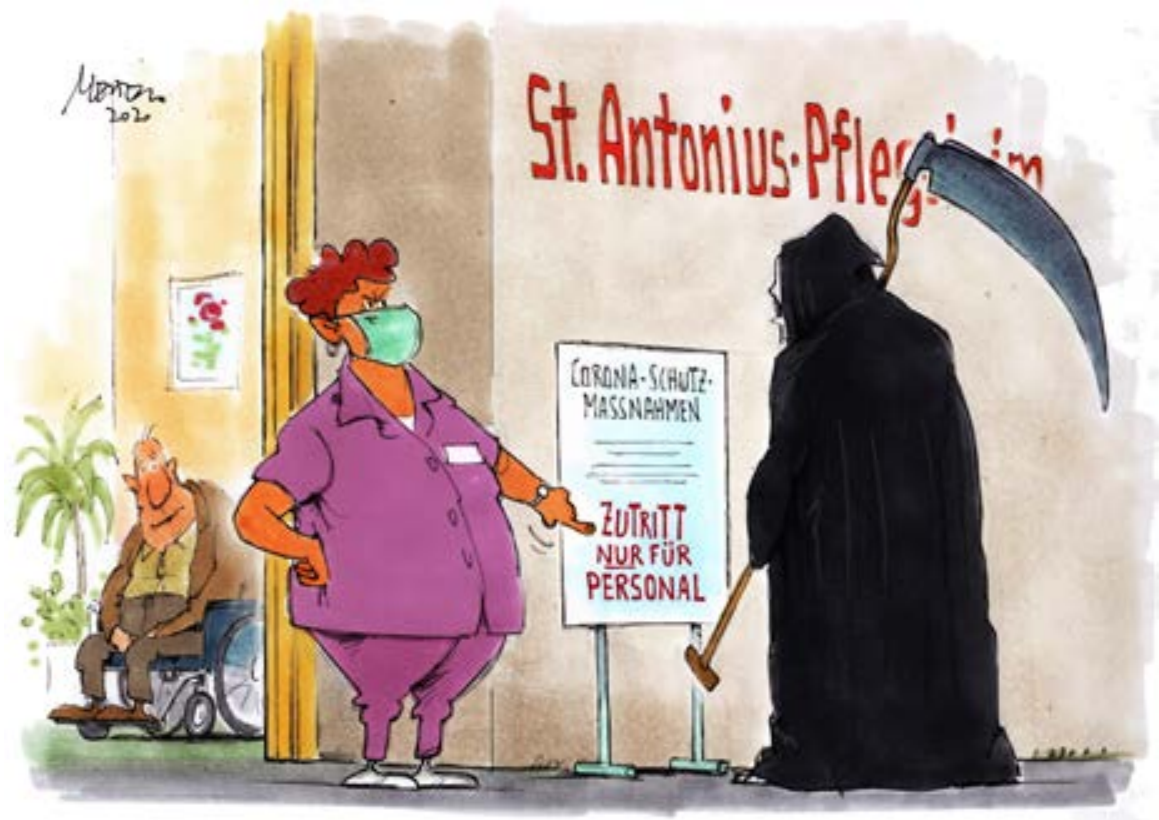


Folgen globaler sozialer Ungleichheiten an die Oberfläche“, wie es Thomas Alkemeyer und Bernd Bröskamp formulieren (76). Beispielsweise haben nicht-weiße Bevölkerungsgruppen in den USA nicht nur eine deutlich größere Wahrscheinlichkeit sich mit dem Virus zu infizieren, sondern leiden durch den erschwerten Zugang zum Gesundheitssystem auch stärker unter den Folgen einer Infektion. „Atemnot, so könnte man sagen, ist für African Americans in den USA der Normalzustand“ (282), wie Jürgen Martschukat in seinem Beitrag herausarbeitet. Der zweite rote Faden sieht die Corona-Pandemie eng mit anderen Krisen verknüpft, insbesondere mit der des Öko- und Klimasystems. Eleonora Rohland betont, dass sowohl Pandemie, als auch die Klimakrise „eine starke Mensch-Umwelt-Verflechtung aufweisen“ (47). Entsprechend zeigen mehrere Beiträge auf, dass die Pandemie mit dem kontinuierlichen Zurückdrängen des natürlichen Wildtierlebensraums im Zusammenhang steht. Gleichzeitig sind die ökologischen Folgen des Lockdowns zunächst positiv, auch wenn sie kaum zu einer nachhaltigeren Postwachstumsgesellschaft führen, wie etwa Klaus Dörre argumentiert (311).

Die Autor`innen verstehen anhand dieser roten Fäden die Pandemie folglich als Vergrößerungsglas für bereits bestehende soziale Probleme. Dazu gehören nicht nur soziale Ungleichheiten und die Klimakrise, sondern auch so unterschiedliche Phänomene wie die „postdemokratische Zementierung der EU“ (Ulrike Guérot, 297) oder „globale Ernährungskulturen im Spannungsfeld von Fragilität und Resilienz“ (Lars Winterberg 331-2). Dieser Blickwinkel lässt sich mit einer Formulierung von Andreas Baier und Christa Müller zusammenfassen: „Krisen machen sichtbar, was vorher schon im Argen lag“ (386).

Des Weiteren bekräftigt eine Reihe von Beiträgen explizit Kontinuitäten mit der Vor-Corona-Zeit. Beispielsweise argumentiert Andreas Reckwitz, das Krisenmanagement in der Pandemie sei ,in keinster Weise ungewöhnlich“, sondern „typisch für die moderne Gesellschaft“ in dem Sinne, dass Staaten bereits zuvor systematisches Management von gesundheitlichen, aber auch technischen und ökologischen Risiken unternahmen (241). Franz Mauelshagen konkretisiert, dass „Ausgangsverbote, Quarantäne, das Herunterfahren wirtschaftlicher Aktivitäten, Versammlungsverbote, selbst das Abschotten ganzer Städte und Regionen, wie in Norditalien, [...] Erbe der europäischen Tradition im Umgang mit Epidemien“ sei (39). Dadurch drängt sich beim Lesen der Eindruck auf, dass die Pandemie qualitativ vielleicht gar keinen Wandel oder Bruch bedeutet, sondern bereits ablaufende soziale Prozesse weiter verfestigt. 


\section{Corona und Protest}

Die meisten Beiträge fokussieren sich auf die gesellschaftlichen Bedingungen, auf die die Pandemie trifft. Darüber hinaus umfasst der Band jedoch auch Kapitel, die sich konkret mit den Protesten gegen die von der Bundes- und den Landesregierungen erlassenen Maßnahmen zur Einschränkung der Pandemie beschäftigen und damit für die Bewegungsforschung von besonderem Interesse sind.

Hervorzuheben ist Christina Henschels Text als einer der wenigen im Band, die sich auf empirische Daten stützen. Die Autorin beobachtete die Straßenproteste in Hamburg gegen die Corona-Maßnahmen zu Beginn der Pandemie und beschreibt dort bereits die wirre Mischung aus vermeintlich unpolitischen Esoteriker*innen, Anhänger*innen von Verschwörungserzählungen, Impfgegner ${ }^{\star}$ innen und organisierten Rechtsextremen, wie sie beispielsweise auch Dieter Rucht (2020) darstellte, und die im Sommer 2020 zu Tausenden in Berlin, Leipzig und anderen Städten zusammengekommen sind. Henschel interessiert sich allerdings weniger für die Zusammensetzung der Demonstrationen, als für die Einordnung von Handlungsrepertoires (siehe dazu auch Mullis 2020). Sie versteht die Proteste „als Akte des physischen, affektiven Präsentwerdens im öffentlichen Raum, als Lockerungsübung im Freeze“ (266) und damit in Kontrast zu Extinction Rebellion und anderen Gruppen der Klimabewegung, die ihre Aktionen als Blockade und Stillstand inszenieren. Gleichwohl könnten diese vermeintlich dynamischen „Lockerungsübungen“ (266) keineswegs emanzipatorisch, sondern als „selbstzentriert und rückwärtsgewandt“ eingeordnet werden (267).

Ingolfur Blühdorn kommt zu einer anderen Einschätzung der Proteste und hält „die von diesen Bewegungen vorgetragene Kritik in verschiedenen Punkten durchaus [für] berechtigt“, kann in ihnen jedoch keine „Bereitschaft zu einer inklusiven, egalitären, deliberativen Politik einer ökologisch und sozial inklusiven Vernunft“ (238) erkennen. Dennoch kritisiert Blühdorn, dass die Proteste „in der öffentlichen Debatte oftmals in toto als irrational zurückgewiesen und als verschwörerische Wahnvorstellung denunziert“ (238) würden. Diese Sichtweise sei „nicht weniger polarisierend und ausgrenzend als die Rhetorik der Rechtspopulisten, von der sie sich absetzen will“ und diene „der Legitimation des Programms zur weiteren Sicherung der Nicht-Nachhaltigkeit“ der Politik (239). Diese Gleichsetzung der Proteste und ihrer Kritiker irritiert nicht nur im Rückblick auf die weitere Entwicklung der Proteste sehr, schließlich waren Verschwörungserzählungen und demokratiefeindliche Narrative von Beginn grundlegendes Kennzeichen der Anti-Lockdown-Demonstrationen, wie Christine Hentschels Beitrag aufzeigt. Darüber hinaus lässt Blühdorn die Frage unbeantwortet, wie mit Corona-Leugner*innen, die sich ganz offensichtlich in ihren eigenen Realitäten bewegen, diskursiv umgegangen werden soll. 
Frank Adloff hingegen versteht die Corona-Proteste als Reaktion auf die „dramatische Kontingenzerfahrung“ (146) der Pandemie. Bei den Demonstrationen gehe es nicht nur um konkrete Einschränkungen, sondern auch darum, mit der Pandemie selbst fertig zu werden. Bei den diffusen und bisweilen menschenfeindlichen Verschwörungserzählungen der Teilnehmer^innen „geht es stets auch um die Wiedergewinnung von kognitiver Gewissheit, Orientierung und affektiver Sicherheit“ (146). Diese Einschätzung wird inzwischen durch eine empirische Untersuchung von Nachtwey, Schäfer und Frei (2020) bekräftigt. Deren Preprint interpretiert „Corona-Dissidenz als Ausdruck einer fundamentalen Legitimationskrise der modernen Gesellschaft“ (61), welche bei den Protestierenden ihre „normative Anziehungskraft eingebüsst“ (62) habe.

\section{Publizieren in der Pandemie}

Auch wenn sich die skizzierten Beiträge spezifisch mit den Protesten gegen die Corona-Maßnahmen auseinandersetzen, so liegt der Mehrwert des Bandes wie eingangs erwähnt vor allem darin, ein breites Panorama der verschiedenen kultur- und sozialwissenschaftlichen Perspektiven auf die Pandemie zu liefern. Allerdings liegt der Fokus der meisten Beiträge darauf, was die Pandemie über bestehende soziale Probleme aussagt. Obwohl die meisten Autor^innen die Corona-Krise als Zäsur verstehen, mag keiner der Beiträge so recht wagen, die spezifischen Charakteristika der im Titel des Bandes anklingenden „CoronaGesellschaft“ zu benennen. Darüber hinaus weisen einige Beiträge inhaltliche und analytische Überschneidungen auf, anstatt derer sich der Rezensent mehr Tiefe in den Analysen gewünscht hätte.

Diese Schwächen des Sammelbandes sind wohl in erster Linie dem frühen Veröffentlichungszeitpunkt geschuldet. Im Juli 2020 lagen nur wenige empirische sozialwissenschaftliche Studien vor und der weitere Verlauf der Pandemie schien ungewiss. Diese Problematik wird von vielen Autor*innen offen thematisiert und veranlasst einige wohl, sich auf das vertraute Terrain etablierter Begriffe und Theorien zurückzuziehen.

Abschließend sei erwähnt, dass inzwischen einige Studien vorliegen, die versuchen, diese Lücken zu adressieren und die Corona-Gesellschaft auch empirisch zu erfassen. Als Beispiel aus der Bewegungsforschung lässt sich etwa die oben erwähnte Baseler Studie (Nachtwey/Schäfer/Frei 2020) nennen, aber auch Artikel zum Schrumpfen zivilgesellschaftlicher Handlungsspielräume durch den Lockdown (Bethke/Wolff 2020) oder zu Handlungsrepertoires in Corona-Zeiten (Pressman/Choi-Fitzpatrick 2020). Inwiefern die Pandemie tatsächlich vor allem bereits bestehende soziale Probleme verstärkt oder ob sie nicht doch transforma- 
tive (progressive wie regressive) Konsequenzen entfaltet, bleibt allerdings abzuwarten. Die Herausforderung einer Gesellschaftsanalyse in Zeiten einer außergewöhnlich dynamischen Krise verdeutlicht vor allem: Die Pandemie ist nicht nur „,unwiderstehlicher Gegenstand“ für die Soziologie, wie Sascha Dickel (78) sie nennt. Sie ist, auch aus sozialwissenschaftlicher Sicht, ein Gegenstand, der sich kontinuierlich weiterentwickelt - so wie das Virus selbst.

\section{Literatur}

Bethke, Felix S./Wolff, Jonas 2020: COVID-19 and Shrinking Civic Spaces: Patterns and Consequences. In: Zeitschrift für Friedens- und Konfliktforschung 9, 363 - 374.

Mullis, Daniel 2020: Protest in Zeiten von Covid-19: Zwischen Versammlungsverbot und neuen Handlungsoptionen. Forschungsjournal Soziale Bewegungen 33(2), 528 - 543.

Nachtwey, Oliver/Schäfer, Robert/Frei, Nadine 2020: Politische Soziologie der Corona-Proteste. Grundauswertung. Universität Basel. SocArXiv Papers, doi.org/10.31235/osf.io/zyp3 f.

Pressman, Jeremy/Choi-Fitzpatrick, Austin 2020. Covid19 and Protest Repertoires in the United States: an Initial Description of Limited Change. In: Social Movement Studies, doi.org/10.1080/14742837.2020.1860743.

Rucht, Dieter 2020: Vereint nur im Unmut. Die Zeit, 25. Mai 2020, zeit.de. 